Cahiers d'études italiennes

\title{
«Le leggi del meraviglioso»: fiaba, novella
}

\section{e romanzo}

"Les lois du merveilleux» : fable, nouvelle et roman

"The Laws of Fantasy": Fairy Tale, Novella and Novel

\section{François Bouchard}

\section{(2) OpenEdition}

\section{Journals}

Edizione digitale

URL: http://journals.openedition.org/cei/3226

DOI: $10.4000 /$ cei.3226

ISSN: 2260-779X

\section{Editore}

UGA Éditions/Université Grenoble Alpes

Edizione cartacea

Data di pubblicazione: 30 décembre 2016

Paginazione: 183-194

ISBN: 978-2-84310-337-7

ISSN: 1770-9571

\section{Notizia bibliografica digitale}

François Bouchard, ««Le leggi del meraviglioso»: fiaba, novella e romanzo», Cahiers d'études italiennes

[Online], 23 | 2016, online dal 23 janvier 2017, consultato il 27 mars 2021. URL: http://

journals.openedition.org/cei/3226 ; DOI: https://doi.org/10.4000/cei.3226 


\title{
«LE LEGGI DEL MERAVIGLIOSO»: FIABA, NOVELLA E ROMANZO
}

\author{
François Bouchard \\ Université François Rabelais de Tours
}

\begin{abstract}
«O cervel balzano da tutti i quattro piè» ${ }^{\mathrm{I}}$, esclama l'oste Biagio dopo aver ascoltato, con altri suoi avventori, la lunga «novella» che, narrata dal tintore Lazzaro, compone la materia stessa del racconto di Francesco Domenico Guerrazzi intitolato La serpicina. Con questa apostrofe, Biagio palesa la propria attinenza al mondo contadino in cui vive, su quel «monte altissimo ${ }^{2}$ dove nevica l'ultimo giorno di carnevale, perché mantiene la presenza testuale della metafora equina («Balzano da quattro, caval da matto", recita il Tommaseo-Bellini) di cui lo stesso narratore fa a meno quando descrive Lazzaro come "un cervello balzano», che "ha il capo pieno di girandole» — cioè, di pensieri stravaganti — , il quale «abbaca sempre co' suoi ghiribizzi e parla per via di parabole» ${ }^{3}$. Così passa di contrabbando, tra le pieghe del testo guerrazziano, la figura manzoniana del poeta come l'intende «il volgo di Milano, e del contado» ${ }^{4}$, evocata avventatamente da Renzo nell'osteria della luna piena, dopo aver alzato per aria il «pane della provvidenza» raccolto sugli scalini della colonna di
\end{abstract}

I. F. D. Guerrazzi, La serpicina, in Id., Scritti, Firenze, Le Monnier, I847, p. 93 (da qui sono tratte anche le altre citazioni del testo a seguire). Secondo la testimonianza dell'autore, La serpicina sarebbe stata scritta durante il confino di Guerrazzi a Montepulciano nel ı830, in seguito alla sua lettura dell'orazione commemorativa di Cosimo Delfante, il i9 giugno dello stesso anno, nell'ambito dell'Accademia Labronica (cfr. F. D. Guerrazzi, Cosimo-Damiano Delfante, in Orazioni funebri di illustri italiani, Firenze, Le Monnier, $1848^{3}$, pp. 45-78). Il testo viene pubblicato per la prima volta nel volume degli Scritti del I847, accanto a un altro inedito, I Nuovi Tartuffi (cfr. C. Biagioli, L'«opera d'inchiostro". Storia editoriale della narrativa di Guerrazzi [1827-1899], Firenze, Editrice Fiorentina, 2006, pp. 93-95).

2. F. D. Guerrazzi, La serpicina, cit., p. 7I.

3. Ivi, p. 77 .

4. A. Manzoni, I Promessi sposi, S. S. Nigro (a cura di), Milano, Mondadori, «I Meridiani», 2002, t. II, p. 28I. 
san Dionigi's: «un cervello bizzarro e un po' balzano, che, ne' discorsi e ne' fatti, abbia più dell'arguto e del singolare che del ragionevole» ${ }^{6}$.

E nei fatti ha senz'altro del singolare, il tintore Lazzaro, non fosse altro che per la prevalenza nel suo aspetto del colore turchino: turchine le calze, "turchini i calzoni; la veste, la sottoveste, la camicia turchine; turchine le mani, ed anche la faccia turchina» 7 : un turchino così pronunciato, e così equamente distribuito sulla sua persona, tra pelle e vestiti, da trasformarlo in possibile insegna pubblicitaria per la «mostra d'indaco» ${ }^{8}$ di un droghiere, mentre il suo naso «infuocato e cremesino" evoca al narratore che lo incontra "sul far del vespro» «la spada fiammeggiante del Cherubino posto a guardia del paradiso terrestre» ${ }^{9}$. Anche con un fisico così singolare da esporlo agli sguardi di tutti — sia allo sporto dell'umile bottega che davanti al giardino dell'Eden —, Lazzaro sceglie di esprimersi, se non proprio per mezzo di una parabola (come annuncia il narratore), almeno attraverso una favola, pretendendo di illustrare il detto non fare mai bene se non vuoi avere del male ${ }^{\mathrm{IO}}$.

Ma la sua è una favola ambigua che, nonostante abbia l'intento moralistico dell'apologo, nonostante voglia «insegnare più che intrattenere»" - e bisogna saper intrattenere per narrare a vanvera in un'osteria mantiene le caratteristiche del racconto, con un andamento narrativo che la riallaccia alla fiaba. Per di più, in essa "ci troviamo dinanzi a esseri fantastici: non personaggi che agiscono effettivamente nel racconto, bensì esecutori dell'evento etico» ${ }^{12}$, come il Gatto con gli stivali di Charles Perrault di cui parla André Jolles:

5. A questo pane si ricollega la farina che Renzo scorge subito dopo, sugli abiti e nei tratti dei componenti della «trista famiglia» incontrata all'imbocco della città: infarinati tutti e tre, e carichi degli avanzi del saccheggio, in mezzo ai quali si staglia il sacco di farina "bucato qua e là» che regge l'uomo. E a quello spreco di farina si appoggia Riccardo Ceroni per tornare a sua volta ad addurre i poeti, quelli veri, e le loro manchevolezze: «Ti ricordi, ne' Promessi sposi, del sacco, onde usciva, a sprazzi, la farina? Un polverio... un'orma bianca... Quanta fatica a raccoglier tanto, da quegli spizzichi, da cuocerne un pane! - Le immagini d'alcuni poeti sono il polverio di quel sacco». (R. Ceroni, Pensieri d'ogni colore, in Frastagli, Milano, Tipografia di Giuseppe Redaelli, I845, pp. I72-I73.)

6. A. Manzoni, I Promessi sposi, cit., p. 28I.

7. F. D. Guerrazzi, La serpicina, cit., p. 75.

8. Ivi, p. 76 .

9. Ibid.

Io. Ivi, p. 77 .

II. «In genere [la favola] è più concreta e breve della fiaba, perché non ha la natura del racconto ma quella dell'esempio. Vuole insegnare più che intrattenere». (G. Ruozzi, Bestie, cose, persone: storie e figure di intrattenimento morale, in Id., Favole, apologhi e bestiari, Milano, BUR, 2007, p. vi.)

I2. A. Jolles, Forme semplici, in Id., I travestimenti della letteratura. Saggi critici e teorici (I897-1932), S. Contarini (a cura di), Milano, Mondadori, 2003, p. 433. 
Egli [il Gatto con gli stivali] è invece lo strumento per riparare l'ingiustizia subita, così che l'animale senza valore, grazie al quale il povero figlio del mugnaio riceve alla fine più di quanto il destino gli abbia riservato, trionfa su un essere [l'orco] che per natura ostacola la legge naturale degli eventi, la felicità ${ }^{13}$.

Nel racconto del tintore Lazzaro, chi assolve a tale mansione nella lite impari che intenta la serpe al montanaro è la scimmia, la quale per prima tra gli animali interpellati si pronuncia a favore dell'umano, impedendo che venga divorato come era stato sentenziato dalla stessa serpicina trasformatasi in mostro ( $\mathrm{Fa}$ l'atto di contrizione, che io voglio mangiarti vivo» ${ }^{14}$ ), nonostante che lui l'avesse precedentemente salvata e assistita. Tanto sicura della "bontà della sua causa» "is risulta la serpe, da proporre all'uomo di scegliere un giudice a suo piacimento per decidere la loro lite. Tre volte si ripete la stessa scena: i due contendenti si avviano in cerca di un giudice in cui, ogni volta, si imbattono in modo del tutto casuale. Prima tocca al cane storpio, guercio e «tanto guasto dalla tigna da disgradarne san Lazzero" ${ }^{16}$ che, senza troppi indugi, si arrende alle ragioni della serpe ("essere d'intuitiva evidenza l'uomo nella sua qualità di uomo meritarsi la morte; per questo perché avendo questa creatura proclamato il diritto di potere mangiare tutti, ognun sentiva che i divorandi nei congrui casi di ragione avevano diritto a mangiare lui $\left.{ }^{17}\right)$ e delibera "di posta» condannando «l'uomo ad essere mangiato vivo» ${ }^{18}$. Ma appellandosi quest'ultimo alla sentenza, e la serpe accondiscendendo nuovamente, i due riprendono a camminare finché non incontrano un diverso giudice, nella figura di «un cavallo che pareva quello dell'Apocalisse, pieno di guidaleschi [...], arrembato, i fianchi sporgenti in guisa da potervi appiccare il mantello» ${ }^{19}$, il quale pronuncia però la stessa sentenza ( Su l'anima di cavallo di garbo, serpe, tu puoi divorarti l'uomo senza un rimorso al mondo» ${ }^{20}$ ). E questo porta di rimbalzo il condannato a un ultimo tentativo per annullare la sentenza di morte invocando la Cassazione, la quale gli viene concessa dalla serpe come «esperimento estremo» ${ }^{21}$, ancorché, come essa fa notare, quest'ultimo grado di giudizio non si usasse nella Toscana granducale.

\footnotetext{
13. Ibid.

I4. F. D. Guerrazzi, La serpicina, cit., p. 8o.

I5. Ivi, p. 8I.

I6. Ibid.

I7. Ivi, p. 82.

I8. Ibid.

I9. Ivi, p. 85 .

20. Ivi, p. 86.

2I. Ivi, p. 88.
} 
Così, scorta una scimmia che coglie fichi, il montanaro la chiama e perora la propria causa evocando la moglie e i figli «che del lungo aspettare si disperano» ${ }^{22}$. Quella scimmia dal canto suo appare così dotta che sembra uscita dalla favola di La Fontaine Le Loup plaidant contre le Renard par-devant le Singe ${ }^{23}$ più che non da quella di Lorenzo Pignotti, La scimia, $o$ sia il buffone $e^{24}$, dove recita la parte del parassita. Infatti, anche se incontrata per strada, la scimmia si mostra stranamente preparata, autorevole e padrona del linguaggio e della prassi giurisprudenziale, e perciò capace di dettare le regole: fino a imporre alla serpe di tornare controvoglia sul luogo del primo incontro tra i due, là dove il montanaro si era convinto a venire in aiuto della serpicina lamentosa e dove poi se l'era trovata trasformata in mostro divoratore. E lì, con una beffa, la scimmia la neutralizza e salva il montanaro.

Tuttavia la morale della favola, che la scimmia conclude con una lunga requisitoria contro il genere umano ( La ferocia umana non trova ferocia che la superi e nemmeno che la uguagli ${ }^{25}$ ), appare ambigua. Con un intento correttivo, e a nome del bene collettivo contro «il malvagio» che quando si vede "tenuto in pregio e premiato» «indura nella nequizia e raduna forze per continuare nella flagellazione delle creature dabbene» ${ }^{26}$, essa arriva addirittura a commentare come ultima ratio che "le serpi non si raccolgono, ma si calpestano». Da generico, il discorso si fa precettistico, per poi scendere a nuovi compromessi che finiscono col promuovere la carità perché appaga la coscienza, «suprema premiatrice dei buoni» ${ }^{27}$, azzerando del tutto l'intento dimostrativo annunciato da Lazzaro intorno alla necessità di non fare mai bene se non vuoi avere del male.

A dispetto di un andamento complesso scandito da più tappe, sia spaziali che narrative, in cui il montanaro si trova a fare i conti con un'animalità dolente, rissosa e vendicatrice, in lotta con un'umanità sopraffattrice e vessatoria, e di una struttura narrativa che accomuna la "parabola» del tintore a una fiaba vera e propria, occorre rilevare l'assenza, nel racconto,

22. Ivi, p. 89 .

23. «Devant le Singe il fut plaidé, / Non point par avocats, mais par chaque partie. / [...] Le juge, instruit de leur malice, / Leur dit : "Je vous connais de longtemps, mes amis, / Et tous deux vous paierez l'amende : / Car toi, Loup, tu te plains, quoiqu'on ne t'ait rien pris; / Et toi, Renard, as pris ce que l'on te demande."” J. de La Fontaine, Le Loup plaidant contre le Renard par-devant le Singe, in Id., Fables, II, 3, Paris, Le Livre de Poche Classique, 2002, p. 90.

24. «Corre la Scimia, e intridesi la faccia, / Poi del tagliante ferro arma la mano; / Ma le gote, e la gola si recide: / Urla il Buffone, ed il Padrone ride». L. Pignotti, La scimia, o sia il buffone, in P. Piranesi (a cura di), Nuova scelta di poesie tratte dai più celebri poeti italiani, Paris, Baudry, I852, p. 173.

25. F. D. Guerrazzi, La serpicina, cit., p. 92.

26. Ibid.

27. Ivi, p. 93. 
di ciò che Jolles ha definito l'oggetto transizionale «impregnato del potere della forma», capace di «rappresentarla allo stesso modo in cui la reliquia rappresenta la forma della leggenda»: ovvero di uno di «quegli oggetti che la fiaba sottrae alla realtà per trasformarli secondo le leggi del meraviglioso" ${ }^{28}$, come la zucca del giardino di Cenerentola che si trasforma in carrozza, o la noce che, in altre fiabe, contiene un abito meraviglioso invece di un gheriglio. Vero è che nel mondo spoglio e ridotto all'essenziale dove si muovono il montanaro e la serpicina compaiono ben pochi oggetti, a esclusione della "grossa pietra" con cui la scimmia imprigiona definitivamente la serpe nel buco dove è stata così ingenua da infilarsi dietro suo suggerimento: un oggetto, quest'ultimo, che d'altro canto pertiene interamente alla realtà e alla sua dimensione più materica, senza nessuna attinenza con il meraviglioso.

Quasi a supplire a tale assenza di marca fiabesca, compare però due volte nella narrazione di Lazzaro la reduplicazione espressiva dell'imperativo cammina cammina, ogni volta nelle brevi sequenze in cui montanaro e serpe mettono la via tra le gambe in cerca di un giudice atto a decider delle ragioni di ognuno. Prima il cane ( Cammina, cammina, ecco farsi incontra a loro un cane che veniva via a scavezzacollo per quanto lo potevano portare tre gambe, che la quarta teneva attratta, come se storpio e' si fosse» ${ }^{29}$ ), poi il cavallo ( $\mathrm{E}$ cammina, cammina, eccoti un cavallo che pareva quello dell'Apocalisse, pieno di guidaleschi, con le saliere sopra gli occhi, arrembato, i fianchi sporgenti in guisa da potervi appiccare il mantello» $\left.{ }^{30}\right)$, mentre la ricerca dell'ultimo giudice si contraddistingue per una brevità ${ }^{3 \mathrm{I}}$ ( $\mathrm{E}$ si rimettono in via; né andarono gran tratto, quando parve loro vedere, e videro certo, qualche cosa che si agitava sopra un albero ${ }^{32}$ ) che giustifica l'elusione dell'imperativo reduplicato nella terza occorrenza della stessa situazione. Come nota Cristiana De Santis, «questa

28. A. Jolles, Forme semplici, cit., p. 434. Riguardo a questo tipo di oggetti, Jolles precisa, nella stessa pagina: «Confesso tuttavia di non aver trovato un nome per elementi oggettivi di questo tipo" (sulla questione ritorneremo più avanti).

29. F. D. Guerrazzi, La serpicina, cit., p. 8I.

30. Ivi, p. 85 .

3I. A sottolineare la brevità, la presenza nella frase della forma del cum inversum o subordinazione inversa segna una accelerazione dei tempi dell'azione, in opposizione all'effetto sortito dall'imperativo reduplicato: «Il rapporto temporale che lega i membri della subordinazione inversa non può dunque essere interamente ascritto alla categoria della contemporaneità né tanto meno a quella, ancora più circoscritta, dell'incidenza: piuttosto la gamma di possibilità comprende un arco che va dall'immediata anteriorità fino all'immediata posteriorità dell'azione dell'apodosi rispetto a quella della protasi». F. Bianco, Il cum inversum fra italiano antico e moderno, in Actes del XXVI Congrés Internacional de Lingüistica i Filologia Romàniques (València, 6-II setembre 20Io), Berlin, De Gruyter, in corso di stampa. Ringrazio Cristiana De Santis per avermi fatto notare la presenza di questa formula.

32. F. D. Guerrazzi, La serpicina, cit., p. 88 
formula durativa [...] suggerisce una durata indeterminata, coerente con la dimensione temporale del racconto fiabesco e al tempo stesso segnala la contrazione del tempo reale all'interno del testo» ${ }^{33}$. Si tratta quindi, con cammina cammina, di figurare il trascorrere di un lasso di tempo lungo e di significare metaletterariamente la dimensione convenzionale di tale rappresentazione dentro la narrazione. Inoltre, poiché l'uso dell'imperativo raddoppiato è prevalentemente riconducibile a una pratica orale della lingua nell'ambito di un'area culturale e linguistica toscana ${ }^{34}$, la sua presenza nel racconto del «turchino» Lazzaro, riferito dal narratore, è perfettamente coerente con la situazione implicita nella comunicazione: in osteria, tra persone di grande dimestichezza, che sono sul punto di rinsaldare il loro legame attraverso il vincolo familiare, previa l'unione dei rispettivi figli.

Dentro la novella di Lazzaro ben pochi sono gli elementi paesaggistici: la strada, il buco dove trova riparo e poi viene imprigionata la serpicina, la siepe che un fossato rende difficilmente raggiungibile dal cavallo, il fico dove armeggia la scimmia; elementi tutti finalizzati allo svolgimento del racconto ma troppo scarni e dispersi per organizzarsi alla stregua di uno scenario vero e proprio. La descrizione rimane molto al di qua della forma di realismo che si dispiega per esempio in una fiaba come Le Petit Poucet, dove, abbandonati nel folto della foresta, i figli dei taglialegna credono di sentire da ogni parte "des hurlements de loups qui venaient à eux pour les manger» in mezzo al vento "qui leur faisait des peurs épouvantables», finché non sopravviene «une grosse pluie qui les perça jusqu'aux os» ${ }^{35}$, per cui i bambini scivolano nel fango. Appena scorta da Pollicino, salito in cima a un albero, "la petite lueur» di una candela, essi debbono ancora arrancare nel bosco e perderla di vista «toutes les fois qu'ils descendaient dans quelques fonds», prima di bussare alla porta della casa "où était cette chandelle» ${ }^{36}$. La fiaba s'immerge in un universo ricollegabile a una comune esperienza del mondo, e bastano pochi elementi a costruire, se

33. C. De Santis, Cresci, cresci, cresci... La reduplicazione espressiva come strumento di espressione di relazioni transfrastiche, in C. De Santis et al., Le relazioni logico-sintattiche. Teoria sincronia diacronia, Roma, Aracne, 20I4, p. I87.

34. «Le ipotesi della toscanità e dell'appartenenza ai registri colloquiali degli imperativi raddoppiati sono avvalorate da un altro indizio, la frequenza di prime attestazioni o attestazioni antiche in autori toscani, quali Giusti o Collodi, o in testi teatrali, per natura mimetici del parlato, quali quelli di Goldoni». A. M. Thornton, Imperativi raddoppiati: un tipo di converbi, in A. Ferrari (a cura di), Sintassi storica e sincronica dell'italiano. Subordinazione, coordinazione, giustapposizione, Atti del X Congresso della Società Internazionale di Linguistica e Filologia Italiana (Basilea, 30 giugno-3 luglio 2008), Firenze, Franco Cesati, 2009, vol. II, p. II96. 35. Ch. Perrault, Le Petit Poucet in Id., Contes, texte établi, présenté et annoté par T. Gheeraert, Paris, Honoré Champion, 20I2, p. 247.

36. Ibid., p. 248. 
non proprio un paesaggio, almeno la precisa percezione che i protagonisti hanno dell'ambiente in cui sono stati repentinamente precipitati dalla fuga dei genitori in un "faux-fuyant». Così il bosco a loro familiare torna a popolarsi di lupi, la cui realtà oggettiva rimane ipotetica ${ }^{37}$; e per sfuggire a quella minaccia plausibile ma non attestata, i bambini vanno a buttarsi tra le grinfie dell'orco, pericolo del tutto reale che si spera però di allontanare impietosendo il mostro divoratore ${ }^{38}$. Qui compare lo stile di Perrault, che «ha ascoltato i racconti, li ha compresi nel loro carattere traducendoli poi nella lingua scritta». E, come precisa Jolles, «ingentilisce i passi più ruvidi e colma le lacune, senza tuttavia lasciarsi sedurre dalle divagazioni e dalle descrizioni implicite nella rielaborazione» ${ }^{39}$. L'autore riesce così a imbastire un episodio la cui eco del tutto reinterpretata risuonerà ancora - mediante la traduzione collodiana del volume dei Contes de fées ${ }^{40}$ nel I876 - nel sesto capitolo delle Avventure di Pinocchio, durante la «nottataccia d'inferno» in cui si scatenano gli stessi elementi climaterici, ma secondo modalità che vengono oggettivate da una narrazione prettamente descrittiva, prima addirittura che il burattino abbia da affrontarli e ne venga agito ${ }^{41}$. Tutt'altra impostazione ha l'episodio della fuga di Renzo verso l'Adda nel capitolo XVII dei Promessi sposi, descritto da Domenico De Robertis come «la favola di Renzo" perché

della favola non ci sono soltanto le movenze, ma gl'ingredienti: la fuga, la meta da raggiungere, la notte, il bosco, la paura del bosco, la paura di sé, dei propri passi, le gambe che vogliono correre e non si sentono di correre più, la stessa scoperta, quando tutto sembra perduto, della meta desiderata, il fiume che ha voce, che manda il suo richiamo, la stessa funzione risolutrice del giorno ${ }^{42}$.

37. «Ils croyaient n'entendre de tous côtés que des hurlements de loups qui venaient à eux pour les manger» (ivi, p. 247).

38. "When the French versions of Tom Thumb and Hansel and Gretel knock at the doors of mysterious houses deep in the forest, the wolves baying at their backs add a touch of realism, not fantasy». R. Darnton, Peasants Tell Tales: The Meaning of Mother Goose, in Id., The Great Cat Massacre and Other Episodes in French Cultural History, New York, Vintage Books, 1985, p. 37.

39. A. Jolles, La fiaba nella letteratura occidentale moderna, in I travestimenti della letteratura, cit., pp. I55-I56. 40. Rimando su questo punto alla mia Introduzione ai Racconti delle fate di C. Collodi (I racconti delle fate e Storie allegre, F. Bouchard [a cura di], Edizione Nazionale delle opere di Carlo Lorenzini Collodi, Pescia, Firenze, Fondazione Nazionale Carlo Collodi, Giunti, vol. IV, 2015, pp. 23-35).

4I. «Per l'appunto era una nottataccia d'inferno. Tonava forte forte, lampeggiava come se il cielo pigliasse fuoco, e un ventaccio freddo e strapazzone, fischiando rabbiosamente e sollevando un immenso nuvolo di polvere, faceva stridere e cigolare tutti gli alberi della campagna». (C. Collodi, Le avventure di Pinocchio. Storia di un burattino, R. Randaccio [a cura di], Edizione Nazionale delle Opere di Carlo Lorenzini Collodi, Pescia, Firenze, Fondazione Nazionale Carlo Collodi, Giunti, vol. III, 20I2, p. 78.)

42. D. De Robertis, La favola di Renzo (Promessi sposi, XVII), in Id., Gli studi manzoniani, I. Becherucci (a cura di), Firenze, Franco Cesati, 2014, p. I85. 
In questa «favola», che sarebbe in realtà più opportuno definire come fiaba, il protagonista si trova a sua volta precipitato in un mondo interamente nuovo, e per lui del tutto capovolto, a cui deve per forza sentirsi estraneo per il suo recente statuto di eslege, espulso dal consorzio umano in cui si riconosceva fino al giorno precedente. Passato dallo statuto di vittima a quello di criminale, l'innocente perseguitato di ieri si trova ridotto a semplice fuggitivo, costretto da Gorgonzola in poi a scansare i suoi simili come altrettanti potenziali nemici, dacché «tutto gli faceva ombra» ${ }^{43}$. Nel mondo alla rovescia che è diventato per Renzo il territorio del ducato di Milano, diversamente da Pollicino e i suoi fratelli egli s'ingegna a rifuggire dagli altri, sia viandanti che abitanti del contado, nel suo camminare alla cieca verso l'Adda e la salvezza. Il suo percorso si svolge a ritroso dalla città sconvolta dal tumulto di San Martino fino ai confini estremi dello stato, attraverso una serie di paesi in cui Gorgonzola fa anche da spartiacque fra il giorno e la notte, che lui accoglie come coltre protettiva. Lasciata la strada maestra, Renzo percorre una viottola nelle tenebre ospitali, tra impannate rischiarate da pochi lumicini e troppi latrati dietro le porte delle cascine, e gelsi, viti e «altri segni di coltura umana», i quali "pareva quasi che gli facessero una mezza compagnia» ${ }^{44}$. Man mano che s'allontana del tutto dall'abitato, la campagna coltivata e addomesticata si trasforma progressivamente: prima in "una sodaglia sparsa di felci e di scope», e poi in "macchie più alte, di pruni, di quercioli, di marruche» 45 con la comparsa di qualche albero, finché, seguendo quella straducola diventata sentiero, Renzo non si accorge «d'entrare in un bosco» ${ }^{46}$. E vincendo «un certo ribrezzo» s'inoltra in quel nuovo ambiente dove la luna proietta sul sentiero «l'ombra delle cime leggermente agitate», mentre gli danno noia gli alberi che "gli rappresentavan figure strane, deformi, mostruose» ${ }^{47}$.

L'addentrarsi nel folto del bosco, nel luogo arcaico per eccellenza che sfugge all'incivilimento, per uscirne "sull'orlo d'una riva profonda» da cui vede «'acqua luccicare e correre» ${ }^{8}$, segna la fine di un percorso avviato sul limitare della città e che si snoda quasi fino alla fine attraverso un paesaggio pienamente addomesticato, popolato, sparso di case e di paesi, solcato da strade, straducole e viottole, plasmato dall'attività umana che si manifesta con la coltura tra l'altro di vite, di gelsi e quindi di bachi da seta:

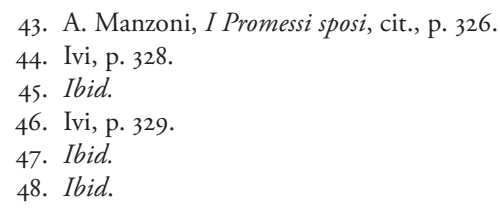


un territorio che pertiene alla seconda natura nella classificazione operata da Iacopo Bonfadio nel I54I, quando aveva scritto, a proposito dei giardini delle ville in riva al lago di Garda, che «la industria de" paesani ha fatto tanto, che la natura incorporata con l'arte è fatta artefice, e connaturale de l'arte, e d'amendue è fatta una terza natura, a cui non saperei dar nome» ${ }^{49}$.

A ben guardare, la determinazione di questa terza natura, quella dei giardini, che si riferisce a un intervento di tipo estetico, del tutto avulso da ogni precisa necessità pratica o economica, si articola sulla seconda natura forgiata e plasmata dall'agricoltura, lo sviluppo urbanistico, le reti viarie e le infrastrutture ${ }^{50}$; la quale a sua volta si riallaccia alla prima natura, quella che risulta in apparenza grezza e incontaminata ${ }^{51}$. Secondo queste categorie, la fuga di Renzo verso il confine - verso il fiume che lo segna e lo incide sulla terra che lui calpesta - si traduce in un passaggio progressivo dalla sconfinata seconda natura che lui sperimenta in tutte le sue gradazioni (città, borgo, paese, aperta campagna) fino alla prima natura che gli si dischiude pregna di minacce indistinte nonostante porti una promessa di salvezza. E mano a mano che egli si inoltra nella sterpaglia dove pruni e cespugli danno adito ad alberi sempre più fitti, mano a mano che vengono meno i segni di coltura umana, Renzo si lascia riportare nella solitudine repentinamente sentita a un passato ormai remoto, accantonato dall'infanzia, e che fa ritorno quando "nella sua mente cominciavano a suscitarsi certe immagini, certe apparizioni, lasciatevi in serbo dalle novelle sentite raccontar da bambino" ${ }^{52}$. Anche aiutandosi con la rassicurante litania "dell'orazioni per i morti», egli deve fare i conti con il precipitarsi delle emozioni mentre s'inoltra nel bosco, dall'uggia e dall' "orrore indefinito" al terrore che lo atterrisce e contro cui deve richiamare «al cuore gli antichi spiriti» ${ }^{33}$. Renzo si trova a contatto con «il silenzio, il vuoto, la solitudine, le tenebre, ed anche più le idee di morte e di annientamento", in un ambiente che sa di sublime, e predispone al dispiegarsi «della forza creatrice» ${ }^{54}$ del poeta. Ma ben poco poeta è qui Renzo, che, ricredutosi

49. I. Bonfadio, Lettera a Plinio Tomacelli, in Id., Le lettere e una scrittura burlesca, A. Greco (a cura di), Roma, Bonacci Editore, 1978, p. 96.

50. "This second nature is what today we would call the cultural landscape: agriculture, urban developments, roads, bridges, ports, and other infrastructures». J. D. Hunt, Greater Perfections. The Practice of Garden Theory, Philadelphia, University of Pennsylvania Press, 2000, p. 33.

5I. «By implication, the first nature becomes for Bonfadio the territory of unmediated nature, what today we might (provisionally and awkwardly) call wilderness". (Ivi, p. 34.)

52. A. Manzoni, I Promessi sposi, cit., p. 328.

53. Ivi, p. 329.

54. Scrive Martignoni nel suo trattato del I8Io, a proposito del sublime: «Convengo perciò volentieri col ricordato Burke nel ritenere, che tutte le privazioni generali sieno grandi, come lo sono infatti il silenzio, il vuoto, la solitudine, le tenebre, ed anche più le idee di morte e di annientamento, siccome quelle, dalle quali 
dalla lezione inflittagli allo spuntar del giorno dai birri, si è dimenticato della figura evocata di sfuggita in osteria tra un bicchiere e l'altro, e adesso ricava dalla sublimità della circostanza solo la forza di stare a sentire finalmente il "mormorio d'acqua corrente»"s dell'Adda.

Così si chiude provvisoriamente la quête di Renzo che trova la salvezza nel cuore profondo del bosco, là dove viceversa nella fiaba di Perrault le Petit Poucet e i suoi fratelli sembrano destinati a perdersi del tutto. In omaggio alla fiaba, la Quarantana dispiega nel filo della narrazione la stessa forma reduplicativa dell'imperativo usata dal tintore Lazzaro nella Serpicina, e questo fin dall'inizio della fuga di Renzo fuori dalle mura di Milano. Appena egli ha varcato la porta orientale, appena entra in una viottola "per evitare la strada maestra», compare la prima occorrenza dell'espressione usata dalla voce narrante: «Cammina, cammina; trova cascine, trova villaggi, tira innanzi senza domandarne il nome; è certo d'allontanarsi da Milano, spera d'andar verso Bergamo; questo gli basta per ora» ${ }^{56}$. Siamo proprio all'inizio del percorso che lo porterà attraverso il paesaggio lombardo, con la sosta all'osteria di Gorgonzola, fin sul limitare della sodaglia, tra seconda e prima natura («Cammina, cammina; arrivò dove la campagna coltivata moriva in una sodaglia sparsa di felci e di scope ${ }^{57}$ ), tappa successiva verso la meta agognata che lui intravede nel cambiamento di vegetazione.

Dal canto suo, Renzo usa la stessa forma reduplicativa nell'avviare il monologo che, cominciato come riflessione sulla strada da prendere, riecheggia la frase d'esordio del viaggio («Verso Milano, non vo di certo») e considera la meta da raggiungere («dunque vo verso l'Adda. Cammina, cammina, o presto o tardi ci arriverò» ${ }^{88}$ ), prima di prendersela con il mercante e le «storie» da lui riferite nell'osteria di Gorgonzola. Il discorso del protagonista, per la sua estraneità alla narrazione, non è in questo caso direttamente ricollegabile alla fiaba, come lo sono invece le altre due occorrenze di "cammina, cammina» che compaiono nella Quarantana assieme all'accenno esplicito alle "novelle sentite raccontar da bambino» (le quali, nella Ventisettana, erano ancora, in modo generico, "cento

l'anima rifugge sbigottita. E a dir vero cotali idee invadono gli animi di un solenne e sacro terrore, il quale tanto più diviene energico, quanto che da nessun limite è frenato l'entusiasmo della commossa fantasia, e lo spirito ripiegato in se stesso tutta dispiega la sua forza creatrice.» I. Martignoni, Del bello e del sublime. Libri due, A. Brettoni (a cura di), Roma, Bulzoni, 1988, p. 73.

55. A. Manzoni, I Promessi sposi, cit. p. 329. Proprio la percezione dello scorrere dell'acqua dell'Adda sembra sciogliergli il sangue, che Renzo si sente allora «scorrer libero e tepido per tutte le vene».

56. Ivi, pp. 3II-3I2.

57. Ivi, p. 328.

58. Ivi, p. 326. 
storie udite» ${ }^{59}$, senza nessuna allusione all'infanzia né alle categorie del genere fiabesco). Viceversa, il passaggio da storia a novella rimanda sia alle «novelle da contare a veglia» che sono "favole ridicole ed inverisimili, come son quelle con le quali si sogliono intrattenere e divertire i fianciulletti» (Tommaseo-Bellini), sia all'uso che numerosi demopsicologi fanno nel secondo Ottocento della parola novella con un significato uguale a fiaba, testimoniate dalle raccolte di Vittorio Imbriani (La novellaja fiorentina, 1871) e di Gherardo Nerucci (Sessanta novelle popolari montalesi, I880): in piena coerenza con la scelta operata da Lazzaro quando chiama "novella» il suo racconto nell'annunciarlo agli amici nell'osteria della Serpicina ${ }^{60}$.

Queste sono le spie testuali che contribuiscono, nella Quarantana, a delineare lo sfondo fiabesco su cui si staglia l'episodio della fuga di Renzo da Milano, il quale acquisisce tale dimensione proprio attraverso il riferimento alle paure infantili suscitate dalle novelle, mentre la presenza della struttura reduplicativa dell'imperativo legata a una matrice orale riporta alla convenzione narrativa della fiaba. Tale dispositivo permette in maniera convincente di «ipotizzare che Manzoni, nel suo intervento correttorio, guardasse a una forma di ripetizione stilizzata, tipica della lingua fiabesca, più che a un generico procedimento espressivo" ${ }^{6 \mathrm{I}}$, cercandovi quindi una efficacia narrativa. Se la pregnanza della reiterazione dell'imperativo, e in special modo della formula specifica "cammina cammina», sembra un tratto distintivo della letteratura fiabesca, anzitutto di matrice toscana, occorre osservare che nella produzione letteraria di genere posteriore alla Quarantana essa non compare, neppure nell'anonima traduzione delle fiabe di Perrault raccolte nel primo dei tre volumi del Gabinetto delle Fate $e^{62}$ pubblicati a Bassano nel I782. E se Collodi, tra gli altri, ricorre spesso a tale formula nelle Avventure di Pinocchio come in altri testi per l'infanzia, egli la usa però un'unica volta nella sua traduzione dei Racconti delle fate, quando, nella Bella e la Bestia di Madame Leprince de Beaumont, rende l'attesa ansiosa del padre della Bella appena arrivato nel castello della Bestia

59. «Pure andò innanzi; e perchè nella sua mente cominciavano a suscitarsi certe immagini, certe apparizioni, lasciatevi in serbo da cento storie udite, egli per discacciarle o per acquetarle, recitava, camminando, e ripeteva preghiere per i morti.» (Ivi, p. 350.)

6o. «Volete voi sentire una novella in proposito? Io ve la racconterò così come so e posso.» (F. D. Guerrazzi, La serpicina, cit., p. 78.)

6I. C. De Santis, Cresci, cresci, cresci. La reduplicazione espressiva come strumento di espressione di relazioni transfrastiche, cit., p. 186.

62. Il primo volume del Gabinetto delle fate (Bassano, Remondini, I782) raccoglie di Perrault le fiabe seguenti: La Bella che dorme nel Bosco, Il picciolo Cappello rosso, La Barba Turchina, Il Gatto Stivalato, Le Fate, Cendrillon, ovvero la picciola Pianella di vetro, Riquetto infioccato e Il picciolo Gallinello, ovvero l'intero volume delle Histoires ou contes du temps passé, nell'ordine originale (cfr. pp. 44-96). 
(«Aspetta, aspetta, e nessuno veniva: finché suonarono le undici e ancora non s'era visto alcuno» ${ }^{63}$ ) con una formula che ricorre quasi uguale nell'episodio dell'impiccagione di Pinocchio ( Ma quando, aspetta aspetta, vide che non compariva nessuno, proprio nessuno, allora gli tornò in mente il suo povero babbo» ${ }^{64}$ ): prima l'attesa del padre che, pusillanime, non saprà sottrarre la figlia alla ferale sorte che avrebbe dovuto spettare a lui; poi l'attesa di un figlio che ricorda il padre quando è ormai svanita ogni speranza di salvezza.

Ma tra La serpicina, la fuga di Renzo verso l'Adda nella Quarantana e le fiabe popolari o letterarie, "gli oggetti che la fiaba sottrae alla realtà per trasformarli secondo le leggi del meraviglioso» (Jolles) sono anche e soprattutto linguistici: accanto alla zucca pronta a diventare carrozza, o alla noce che serra palmi di stoffa finissima, campeggia nella fiaba italiana dell'Ottocento l'imperativo raddoppiato che, dismettendo fiabescamente ogni funzione ingiuntiva, ne acquisisce un'altra, che può essere temporale e/o consecutiva. Cifra della fiaba, di cui palesa la natura idealmente orale, questa struttura si mostra capace di contaminare forme più complesse, come il romanzo, fosse pure nell'ambito di un unico episodio di rilievo, quello manzoniano. Mentre estendendosi a forme meno nitidamente caratterizzate, come il racconto di Guerrazzi e la novella di Lazzaro al suo interno, contribuisce a determinarne la qualità fiabesca. Fiaba o apologo, La serpicina porta in nuce le propaggini di un modello fiabesco che altri, nel secondo Ottocento, avranno difficoltà a sviluppare. Ma questa stessa eterogeneità formale, del resto, pertiene alla forma stessa della fiaba letteraria, come insegna Charles Perrault attraverso il titolo scelto per la sua raccolta delle Histoires ou contes du temps passé, che iscrive sul frontespizio del volume la natura duplice dei racconti tra storia (histoire) ${ }^{65}$, ancorata nel reale, e fiaba (conte).

63. C. Collodi, I racconti delle fate e Storie allegre, cit., p. 244. Il passo traduce la frase seguente: «Il attendit pendant un temps considérable; mais onze heures ayant sonné sans qu'il vît personne, il ne put résister à la faim». Contes de fées tirés de Claude [sic] Perrault, de Mme d'Aulnoy et de Mme Leprince de Beaumont, Paris, Librairie Hachette, 1860 , p. 354.

64. C. Collodi, Le avventure di Pinocchio. Storia di un burattino, cit., p. IIo.

65. Si pensi per esempio a Les Histoires mémorables et tragiques de ce temps di François de Rosset, che, pubblicate la prima volta nel I6I4, sono state regolarmente ristampate fino alla metà del Settecento. 